\title{
The Diffractive Structure Functions from Large Rapidity Gap Data at H1
}

\author{
Paul Laycock* \\ University of Liverpool \\ E-mail: laycock@hep.ph.liv.ac.uk
}

H1 has measured the diffractive DIS cross section $e p \rightarrow e X Y$ using data from both of the HERA data-taking periods. Using new measurements of the diffractive cross section at different centreof-mass energies, the diffractive longitudinal structure function $F_{L}^{D}$ has been extracted at low and medium $Q^{2}$. The results are in agreement with NLO QCD predictions based on fits to inclusive data. New high statistics measurements of the diffractive reduced cross section $\sigma_{r}^{D}$ have been combined with previous $\mathrm{H} 1$ data to produce one coherent diffractive dataset measured over the accessible kinematic range. This precise dataset agrees well with pubished ZEUS data up to a normalisation factor, as well with QCD-based predictions.

XVIII International Workshop on Deep-Inelastic Scattering and Related Subjects

April 19 -23, 2010

Convitto della Calza, Firenze, Italy

\footnotetext{
${ }^{*}$ Speaker.
} 


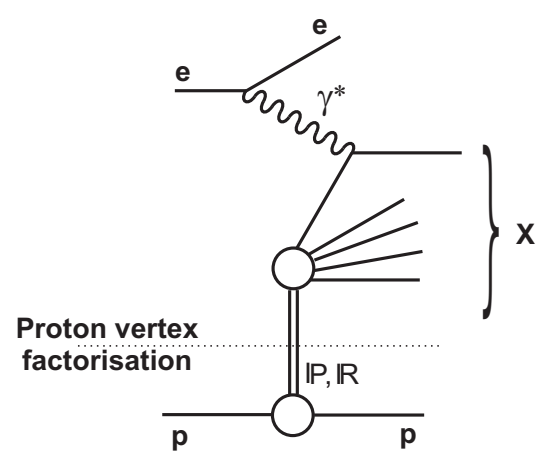

Figure 1: A schematic illustration of the NC diffractive DIS process $e p \rightarrow e X p$ at HERA; the dotted line shows the point at which the diagram can be divided under the assumption of proton vertex factorisation.

\section{Introduction}

It has been shown by Collins [1] that the diffractive DIS process $e p \rightarrow e X Y$ factorises. In Figure 1, an additional assumption is shown whereby the proton vertex dynamics factorise from the vertex of the hard scatter - proton vertex factorisation. Although proton vertex factorisation must be broken in QCD, measurements of the diffractive cross section from both H1 and ZEUS [2, 3, 4] show that it's a good enough approximation to the data such that meaningful next-to-leading order (NLO) QCD fits can be made [2, 5, 6]. Measurements of the dijet cross section in diffractive DIS (DDIS) are particularly sensitive to the gluon diffractive PDF (DPDF) at large fractional momenta, allowing tests of the DPDFs extracted in fits to inclusive data. Such measurements have been used to distinguish between different gluon DPDFs [5]. DDIS events containing charm particles in the final state have similarly been used to test the gluon DPDF [7] at lower fractional momenta.

In analogy with the inclusive case, the DDIS cross section can be expressed in terms of a linear combination of structure functions. In the HERA kinematic regime, this can be well approximated by $F_{2}^{D}$ and a term related to scattering of longitudinally polarised photons $F_{L}^{D}$. The experimental remit is then to extract as much information as possible on these observables in order to provide constraints on models and help elucidate our understanding of diffraction.

\section{Kinematics, Cross Sections and Structure Functions}

The kinematic variables used to describe inclusive DIS are:

$$
Q^{2}=-\left(k-k^{\prime}\right)^{2}, x=\frac{Q^{2}}{2 p \cdot q}, y=\frac{p \cdot q}{p \cdot k} .
$$

Here, $Q^{2}$ is the virtuality of the exchanged boson, $x$ is the Bjorken scaling variable and $y$ is the elasticity. They are defined in terms of $k$ and $k^{\prime}$, the four-momenta of the incoming and outgoing electrons, respectively, and the four-momentum of the incoming proton $p$. In addition to the standard DIS variables and the Mandelstam variables $(t, s)$, the kinematic variable $x_{I P}$ and $\beta$ are useful in describing the diffractive DIS interaction. They are defined as:

$$
x_{I P}=\frac{Q^{2}+M_{X}^{2}-t}{Q^{2}+W^{2}-M_{P}^{2}}, \beta=\frac{Q^{2}}{Q^{2}+M_{X}^{2}-t},
$$


where $M_{X}$ is the invariant mass of the final state hadronic system $X, M_{p}$ is the mass of the proton and $W^{2}=(q+p)^{2}$ is the square of the centre of mass of the photon-proton system. $x_{I P}$ is the longitudinal fractional momentum of the proton carried by the diffractive exchange and $\beta$ is the longitudinal momentum fraction of the struck parton with respect to the diffractive exchange and $x=x_{I P} \beta$. The data are discussed in terms of a reduced cross-section, $\sigma_{r}^{D(3)}\left(\beta, Q^{2}, x_{I P}\right)$, related to the measured differential cross section by:

$$
\frac{\mathrm{d}^{3} \sigma_{e p \rightarrow e X Y}}{\mathrm{~d} x_{\mathbb{P}} \mathrm{d} \beta \mathrm{d} Q^{2}}=\frac{2 \pi \alpha_{e m}^{2}}{\beta Q^{4}} \cdot Y_{+} \cdot \sigma_{r}^{D(3)}\left(x_{\mathbb{P}}, \beta, Q^{2}\right)
$$

where $Y_{+}=1+(1-y)^{2}$. The reduced cross section is related to the diffractive structure functions by:

$$
\sigma_{r}^{D(3)}\left(x_{\mathbb{P}}, \beta, Q^{2}\right)=F_{2}^{D(3)}\left(x_{\mathbb{P}}, \beta, Q^{2}\right)-\frac{y^{2}}{Y_{+}} F_{L}^{D(3)}\left(x_{\mathbb{P}}, \beta, Q^{2}\right)
$$

The suppression term $y^{2} / Y_{+}$means that a measurement of $F_{L}^{D}$ requires cross section measurements at large values of $y$.

Diffractive events are selected on the basis of a Large Rapidity Gap (LRG) being present, such that the hadronic final state consists of a system $X$ detected in the central $\mathrm{H} 1$ detector which is well separated from a system $Y$, which is an elastically scattered proton or its low mass excitation. As the final state system $Y$ escapes detection, the cross section is integrated over ranges in leading baryon mass $M_{Y}$ and $t$ :

$$
M_{Y}<1.6 \mathrm{GeV},|t|<1.0 \mathrm{GeV}^{2}
$$

\section{The Measurement of $F_{L}^{D}$}

Data from three proton beam energies, $E_{p}=460,575$ and $920 \mathrm{GeV}$, have been used to measure the diffractive reduced cross section at the same $x$ and $Q^{2}$, but different $y$, suggested in [8]. Following a similar procedure to that used for the extraction of $F_{L}$ by $\mathrm{H} 1$ [9], these data have then been used, together with previously published data at $820 \mathrm{GeV}$ [2], to extract the diffractive longitudinal structure function $F_{L}^{D}$. The largest sensitivity to $F_{L}^{D}$ is at the largest $y$, requiring measurements of the scattered positron down to energies of $3.4 \mathrm{GeV}$.

The diffractive reduced cross section is integrated over the $M_{Y}$ and $t$ ranges given in equation 2.5. After this correction, the three data sets agree well within the normalisation uncertainties. In order to extract $F_{L}^{D}$ optimally, the cross sections are normalised to the H1 2006 DPDF Fit B result. As the published data at $820 \mathrm{GeV}$ were included in the analysis of the data used as input to H1 2006 DPDF Fit B, they are already consistently normalised. The resulting measurements of $F_{L}^{D}[10]$ are shown in figure 2. The data agree well with the predictions of Fit A and Fit B.

\section{The Measurement of $\sigma_{r}^{D}$}

Data at the nominal proton beam energy of $E_{p}=920 \mathrm{GeV}$, taken in both the HERA datataking periods, have been analysed to extract the diffractive reduced cross section $\sigma_{r}^{D}$ in as wide a kinematic range as possible. These new, precise data agree well with the published H1 data [2] and have therefore been combined using a simple weighted average method in order to produce 


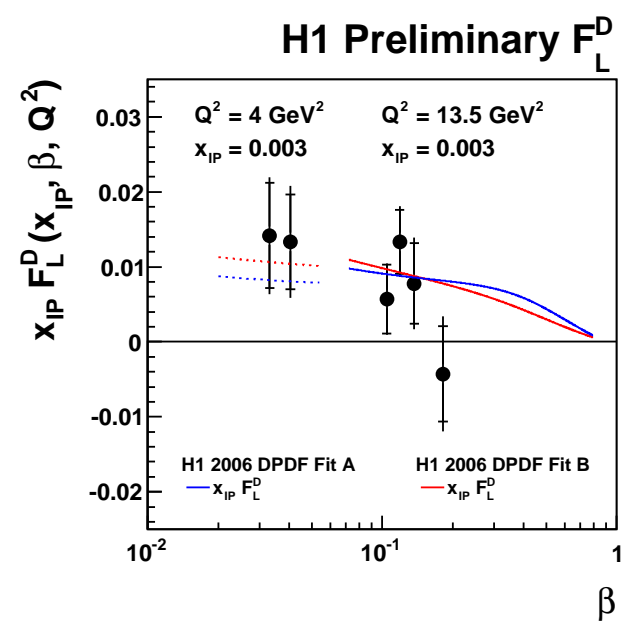

Figure 2: The measurement of $F_{L}^{D}$, multiplied by $x_{\mathbb{P}}$, as a function of $\beta$ for two values of $Q^{2}$. The data are compared to the predictions of Fit A (blue line) and Fit B (red line) with the exprapolations of these fits shown as dashed lines.

a dataset that covers almost the entire accessible kinematic range [11]. The combined data are shown in figure 3 in the two most precise bins of fixed $x_{\mathbb{P}}$. The data are shown as a function of $Q^{2}$ in bins of $\beta$ and are compared to the published ZEUS data [4], which have here been scaled by a factor 0.87 . After accounting for this normalisation difference, which is consistent with the individual normalisation uncertainties, the data agree well throughout the majority of the phase space, although there are differences, in particular at the highest $\beta$. The data compare well with Fit $\mathrm{A}$ and Fit $\mathrm{B}$ and are shown compared to the predicitions of a dipole model [12], which also describes the data well.

\section{Conclusions}

New measurements of the diffractive reduced cross section using data taken at three proton beam energies have been made. The measurements have been combined with existing $\mathrm{H} 1$ data at $820 \mathrm{GeV}$ in order to extract $F_{L}^{D}$ at low and medium $Q^{2}$. The result agrees well with the predictions of Fit A and Fit B. A new measurement of $\sigma_{r}^{D}$ has been combined with the existing H1 published data to produce a combined dataset over nearly the whole accessible kinematic range. The data agree well with published ZEUS data, as well as with models based on QCD.

\section{References}

[1] J. Collins, "Proof of factorization for diffractive hard scattering," Phys. Rev. D 57 (1998) 3051 [Erratum-ibid. D 61 (2000) 019902] [hep-ph/9709499].

[2] A. Aktas et al. [H1 Collaboration], "Measurement and QCD analysis of the diffractive deep-inelastic scattering cross-section at HERA,” Eur. Phys. J. C 48 (2006) 715 [hep-ex/0606004].

[3] A. Aktas et al. [H1 Collaboration], "Diffractive deep-inelastic scattering with a leading proton at HERA,” Eur. Phys. J. C 48 (2006) 749 [hep-ex/0606003]. 

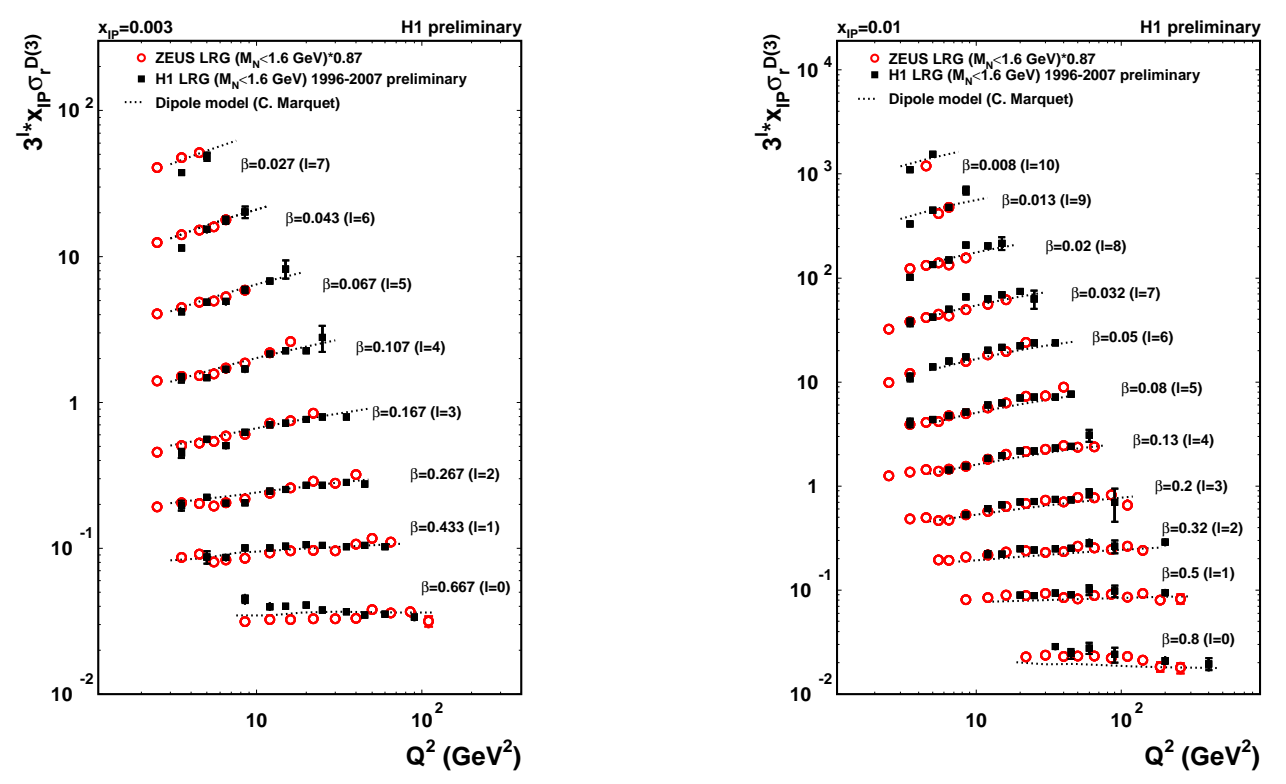

Figure 3: The measurement of $\sigma_{r}^{D}$, multiplied by $x_{\mathbb{P}}$, in two bins of $x_{\mathbb{P}}$ as a function of $Q^{2}$ in bins of $\beta$ The new combined $\mathrm{H} 1$ dataset (black points) are compared to the ZEUS data scaled by 0.87 (red points).

[4] S. Chekanov et al. [ZEUS Collaboration], "Deep inelastic scattering with leading protons or large rapidity gaps at HERA,” Nucl. Phys. B 816 (2009) 1 [arXiv:0812.2003].

[5] A. Aktas et al. [H1 Collaboration], "Dijet Cross Sections and Parton Densities in Diffractive DIS at HERA,” JHEP 0710 (2007) 042 [arXiv:0708.3217].

[6] S. Chekanov et al. [ZEUS Collaboration], “A QCD analysis of ZEUS diffractive data,” Nucl. Phys. B 831 (2010) 1 [arXiv:0911.4119].

[7] A. Aktas et al. [H1 Collaboration], "Diffractive open charm production in deep-inelastic scattering and photoproduction at HERA,’ Eur. Phys. J. C 50 (2007) 1 [hep-ex/0610076];

S. Chekanov et al. [ZEUS Collaboration], Nucl. Phys. B 672 (2003) 3 [hep-ex/0307068].

[8] P. R. Newman, "Prospects for $F_{L}^{D}$ measurements at HERA-II," arXiv:hep-ex/0511047.

[9] F. D. Aaron et al. [H1 Collaboration], "Measurement of the Proton Structure Function $F_{L}$ at Low x," Phys. Lett. B 665 (2008) 139 [arXiv:0805.2809 [hep-ex]].

[10] H1 Collaboration preliminary results H1prelim-09-011 2009. H1prelim-10-017 2010.

[11] H1 Collaboration preliminary results H1prelim-10-011 2010.

[12] C. Marquet, Phys. Rev. D 76 (2007) 094017 [arXiv:0706.2682]. 